\title{
Media Access Control (MAC) Protocols: An Overview
}

\section{EBELOGU Christopher $\mathrm{U}^{1}$, AMUJO Oluyemi $\mathrm{E}^{2}$, ADELAIYE Oluwasegun $\mathrm{I}^{3}$ and FAKI Ageebee $\mathrm{S}^{4}$}

\author{
Lecturer $^{1-3-4}$ and Research Scholar ${ }^{2}$ \\ ${ }^{1-2}$ Department of Computer Science, \\ University of Abuja, Abuja \\ ${ }^{3-4}$ Department of Computer Science \\ Bingham University
}

Karu, Nassarawa State,

Nigeria

\begin{abstract}
Information Technology has grown rapidly leading to challenges with communicating information with nodes at remote locations. There are many communication media, and certain varieties of wired links such as coaxial cables, where multiple nodes can all be linked to hear each other's transmissions either properly, correctly or with some non-zero probability. Generally, there are two rudimentary ways of sharing such stations or media, which can be the time-sharing, and frequency sharing. This paper tackles the rudimentary question of how such one common communication channel also called a shared medium can be distributed among the distinct nodes with maximum productivity. It analyzes wireless sensor networks and its accompanying technologies sighting their pros and cons. Focusing on time-sharing, we examine methodically two prevalent approaches used in obtaining this which are either by time division (split) multiple access (TDMA) or contention protocols of which both approaches are widely being used in today's systems. It also shows that with proper time of selecting the likelihood exigency of Tame Division Multiple Access's (TDMAs), utilization tends to $1 /$ e (37\%) but also tends to increase collision. To adapt the transmitted message likelihood, a format for calculation was adopted. The idea was to seek to gather to the favorable point of value. Slotted Aloha has twice the utilization of Unslotted-Aloha when the numbers of accumulated nodes grow. The Study also shows that each node will stake transmission within sprinkling fixed number of slots and this is guaranteed when using a related distribution in a finite window, but this is not the case with geometric distribution.
\end{abstract}

Key Words: Aloha, Data Traffic, Frequency Modulation, Networks, Wireless Sensor Networks.

\section{INTRODUCTION}

Protocols capture the "rules of engagement" that nodes must engage, so that they can conjointly obtain effective performance. These sharing schemes define how multiple nodes should control their access to a distributed medium, they are classified as media access (MAC) protocols or multiple access protocols. Contention protocols are called so because the node cope with each other for the medium without organizing in anticipation a schedule that determines what node should transmit when the client should transmit, or a frequency reservation that warranties little or no disturbance/interference [1]. These contention protocols are appropriate for data system sites/networks, which are distinguished by nodes transmitting data in bursts including variable rates. This paper is aimed at discussing the general overview of MAC protocols from various perspectives.

\section{LITERATURE REVIEW}

\subsection{MAC Protocols for Wireless Sensor Networks}

TDMA has a good thing about collision-free medium gain access to. On the other side, it provides clock random oscillation problems and reduced throughput at low lots due to idle slots. The difficulty with TDMA systems are the harmonization of the nodes and adjustment to topology modifications where these altering consequence from the introduction of new nodes, exhaustion 
of battery capacities, broken links because of interference, sleeping schedules of relay nodes, scheduling caused by clustering algorithms. The slot tasks, therefore, should be done regarding such possibilities [2,3]. Nevertheless, it can be difficult to alter the slot assignment within a decentralized environment for traditional TDMA, since all nodes must agree on the slot assignments. In parallel with the common networking structure, Carrier Sense Multiple Access (CSMA) methods have a reduced delay and promising throughput potential at lower traffic loads, which generally is actually the case in cellular/cordless sensor networks. However, additional collision avoidance or collision/impact detection methods should be employed to take care of the collision/impact possibilities. Frequency Division Multiple Access (FDMA) is another scheme that offers a collision-free medium. Though, it brings an additional circuitry requirement to dynamically contact by means of communication the different radio channels. This kind of increases the expense of the sensor nodes, which is from the objective of the sensor network systems [4].

Media Access Control (MAC) protocols that may have been made for typical ad hoc networks have generally focused on the customization of fairness and throughput efficiency, with less emphasis on energy conservation [5]. In sensor networks, energy is often more important than throughput and energy management considerations have a big impact on MAC protocols, idle listening costs about as much energy as transmitting [6]. Yet, the energy constraint is typically considered paramount for wireless/mobile sensor networks, and so many MAC protocols have recently been made that brings themselves specifically for the characteristics of sensor networks. Protocols such as Multiple Access with Collision Avoidance for Wireless (MACAW) eliminates the energy waste caused by colliding packets in wireless networks/systems [7].

Furthermore, enhancements have recently been made to these protocols (e. g., Power Aware Multi-Access protocol with Signaling (PAMAS)) to avoid crosstalk. However, it is often shown that idle power consumption can be of the same order as the transmitting and acquiring power consumption, and if so, can greatly have an effect on the overall power consumption, especially in networks with relatively low traffic rates. As a result, the focus of most MAC protocols for sensor networks is to reduce this idle power usage by setting the sensor radios into a rest state as frequently as possible. Some protocols that try to balance throughput and energy consumption include Sensor Media Access Control (S-MAC), Timestamp-ordered Media Access Control (T-MAC), Berkeley Media Access Control (B-MAC) and WiseMAC. [8], [9]

\subsection{Shared Medium}

2.2.1 Satellite communications: Eventually the first example of a shared-medium network deployed for data communication. The Alohanet in Hawaii was designed by a group led by Prof. Norm Abramson in the 1960s at the University of Hawaii as a way to allow computers in several island talk/communicate by connecting them. Computers on the satellite performed as a switch to provide connectivity between the nodes on the island destinations; A communications satellite works like a relay station that is, the signals sent by the ground stations are picked up by the satellite's recipient antennas, the signals are then filtered, their frequency improved and amplified, and then routed via the broadcast antennas down again to Earth. Although, in some cases the signal is first refined by digital processors on board the satellite, just about all satellites, nevertheless, are 'transparent', such that they retransmit the signal without modifying it, the job of these satellites is simply to deliver the signal exactly to where it is required, and these kinds of satellite networks are still used today in several parts of the world, and they are quite pricey, perhaps the most frequent method to obtain connectivity in the high seas and other remote areas of the world. Furthermore the downlink of these same satellite networks usually runs over a different frequency band from the uplinks, which all share the same frequency band. The various uplinks, nevertheless, should be distributed by different simultaneous communications from the ground channels to the satellite [10], [11].

2.2.2 Unguided networks: This is the most common example of a distributed communication medium, and one that is widely increasing in recognition and acceptance, it uses radio signals. Some good examples include cellular wireless networks system standards like 2G, 3G, 4G, and 5G; and cordless networks like LANs (example 802. 11), the Wi-fi/Wireless standard, and various other varieties of radio-based communication. Transmission broadcast is a crucial property of radio and traditional communication in which waves of signals are spread in all directions, the kind of wave that propagate in the sky mode can travel long distances, and low and medium frequencies can penetrate walls, especially with omni-directional antennas. However, radio and acoustic broadcasts are not perfect because of interference, crosstalk and the occurrence of obstacles on certain paths, so different nodes may effectively acquire different parts of a transmission. This reception is nondeterministic and the fundamental random processes that create bit errors are hard to model [10], [12], [13].

2.2.3 Guided bus networks: Categories of guided bus networks include twisted pair, coaxial cable and fiber optics. A great example of a cabled shared medium are Ethernet, which when it was initially developed and for many years after it was developed still used a shared cable to which multiple nodes could be communicate. Any packet that is being transmitted over the Ethernet, could be heard by all stations linked/connected literally physical to the network, creating a perfect shared transmission broadcast 
medium, therefore, if two or more nodes send packets that overlap in time, both packets ended up being distorted and received in error. [14]

2.2.4 Wireless radio and television: Before data communication, many countries in the world had and still have radio and television broadcast stations. Presently, a comparatively small number of transmitters share a rate of a regularity/frequency range to deliver radio or television content. Since each station was presumed to be active almost all of the time, the natural method of sharing is to give away the frequency range into smaller sub-ranges and allocate each subrange to a station; this is known as frequency division multiplexing, therefore, given the practical significance of these examples, and the sea change in network access that is as a result of wireless technologies, expanding strategies to share a common medium is an essential/ vital issue.[15]

\subsection{Communication Procedures}

Communication between a collection of ground stations and up station takes place by transmitting data from the downlink station up to the one in the sky (uplink) as shown in Fig. 1, frequencies are not shared between the ground station because they do not know how long the downlink will communicate with the satellite. Therefore the downlink have the same frequencies, so if two ground stations communicate up at the same time, the satellite will probably not able to take together both different transmission or signal apart as they are on the same frequency, this may therefore lead to collision of the data being dispatched, although this could still be done by using Frequency Division Multiplexing (FDM), but they do not because, if one satellite has data to transmit and the other does not, frequency will be wasted. Pertaining to the communication between nodes without collision, wait or waste of frequencies, the key goal for MAC standard protocol includes the avoidance of collision between transmissions, which happens when transmissions are concurrent, thereby producing a communication protocol/rules of engagement that ensures good performance[10]-[11],[4], [16].

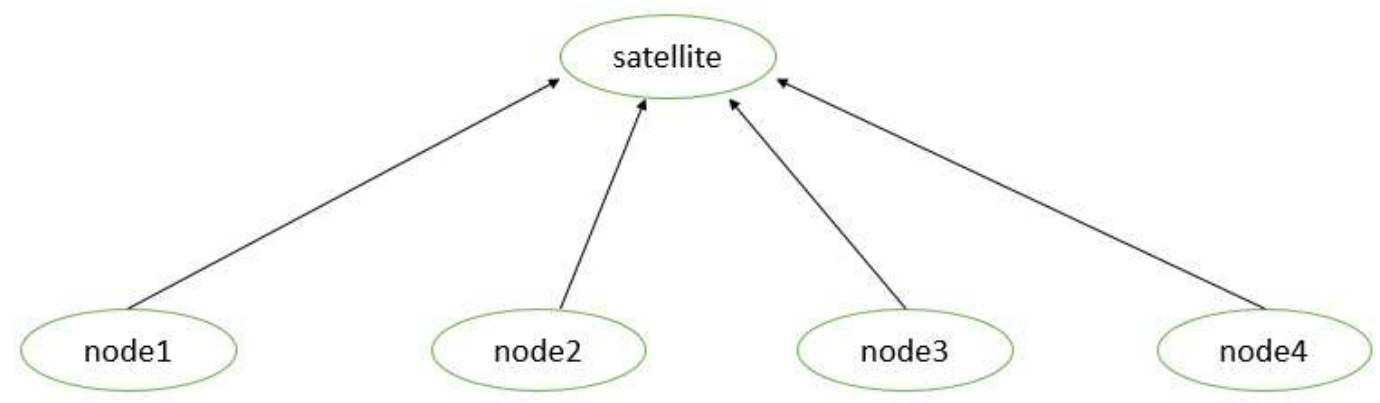

Fig 1: Communication between up station and ground stations

There are three (4) different ways to solving this problem

1. Frequency Sharing (FDM): To use FDM, just by allocating frequencies to any node, but we do not because if frequency is allocated to an empty queue in a node then the frequency that is supposed to be used by a node with packets will be wasted. Characteristics of FDM includes:

- $\quad$ assign a certain frequency to a transmission channel

- $\quad$ permanent (radio broadcast), slow hopping (GSM), fast hopping (FHSS, Frequency Hopping Spread Spectrum)

2. Time Sharing (TDM): to divide up time amongst the nodes, in this case scenario each nodes gets access to the satellite for some fraction of time.

3. Contention Protocols (CSMA): Contention protocols like Aloha and CSMA do not experience these problems, but unlike TDMA, they face packet collisions. Generally, data burst and skewed workloads favor contention protocols over TDMA, the intuition behind these protocols is that we somehow want to designate access to the medium fairly, but only among the list of accumulated nodes. Unfortunately, only each node knows with guarantee if it is accumulated or not, the solution therefore is by using randomization, a simple but really powerful idea, but if each accumulated client (backlogged) transmits data with some probability, perhaps nodes can then be organized to pick their transmitting probabilities to achieve an outcome that yields sensible and 'fair' utilization (throughput) and justness, such as a node or set of nodes transmitting signal when they really feel like transmitting signals or packets, retry if packets from different nodes collide with one another, minimize the collisions between nodes, provides additional booking modes, and solves the challenge of the receiver being awake almost all the time to receive signals as well

4. Scheduling Protocols (TDMA): Scheduling protocols uses a "pre-computed" schedule to transmit messages, they are also distributed, and adaptive solutions are difficult to achieve [2]-[3], [5], [7].

\subsection{Matrix of MAC Protocol}


The matrix is developed to achieve a good protocol that handles "data failure". The requirements of contention protocol to effect the solution to the data failure problem is defined below;

1. Throughput: which is measured in bits/sec or packet/sec, is the amount of work that can be performed at a given time, the throughput of MAC protocol requires utilization which is measured in percentage and can be defined by dividing the throughput that a protocol achieves by the maximum rate of data that is sent on that channel. This can be calculated mathematically as:

$\mathbf{U}(\mathbf{0 , 1})=\frac{\text { Throughput }}{\text { maxrate }}$, the higher the utilization $\mathrm{U}$ the higher the throughput

Example: if you have four (4) nodes with maximum rate 10bps and the throughput of each set are: $1 \mathrm{mps}, 4 \mathrm{mps}, 2 \mathrm{mps}$, and $1 \mathrm{mps}$. What is the utilization?

Solution: $U=8 / 10=0.8$, which is between 0 and 1 .

2. Fairness index: in this case scenario the utilization and throughput doesn't matter, amongst N nodes, the probability of everybody getting equal fairness is 1 , this is calculated mathematically as:

f\{0 and 1$\}=\left(\frac{\left.\sum x i\right) 2}{\mathrm{~N} \cdot \sum x \boldsymbol{i} 2}\right)$, where $x i$ is the throughput and $\mathrm{N}$ is the number of nodes.

One node hoarding between two nodes is fair compared to one node hoarding amongst five nodes.

3. Delay: a very high throughput and fairness can be gotten but with delay, the performance characteristics is decreased, therefore the bonded delay would be integrated which measured by when the packet was in the queue till when it got delivered.

$[17-18]$

\section{5 . Contention Protocol (MAC)}

\subsubsection{Pure Aloha (Unslotted)}

This was developed by Prof. Norm Abramson in Hawaii, who created this scheme to connect islands via satellite networks. Pure aloha is simpler, although expensive, with no synchronization, however, collision probability increases because there is a factor-2handicap of unslotted vs. Slotted.

There are two assumptions:

- $\quad$ Packets can only be transmitted at the beginning of a time slot.

- $\quad$ Every packet is an integer number of time slots

[19][20]

\subsubsection{Slotted Aloha}

In slotted aloha, time is slotted, that is packets cannot be sent at an arbitrary point in time unto the network, instead time is divided into time slots, this slots could be any length which does not matter. We assume that the stations are perfectly synchronous, in each time slot each station transmits with probability $p$ [21].

Suppose there are $\mathrm{N}$ backlogged nodes and each node uses the same outlay of $\mathrm{p}$. We can earlier calculate the strain of the shared medium as a function of $\mathrm{N}$ and $\mathrm{p}$ by practically counting the various numbers of slots in which unquestionably one node sends a packet. By definition, a one slot by the whole of 0 or in a superior way than 1 transmissions does not satisfy in a satisfactory manner of delivered packets, and appropriately does not partake toward the utilization therefore if each node sends mutually eventuality $p$, then sure as can be that one node sends in any packets if and only if slot is $\llbracket N p(1-p) \rrbracket \wedge(N-1)$. The purpose is that the fortuity that a specific node sends packets in the time slot is $\mathrm{p}$, and for its transmission to be established, most part the distinctive nodes should not transmit packets/waves/signals, the corporate probability is $\llbracket p(1-\mathrm{p}) \rrbracket \wedge(\mathrm{N}-1)$. We can pick the smoothly transmitting node in $\mathrm{N}$ different ways, therefore the probability of unconditionally one node transmitting in a slot is 『 $\mathrm{Np}(1-\mathrm{p}) \rrbracket \wedge(\mathrm{N}-1)$, this degree is the utilization achieved by the contention protocol because it is the bit fractions of slots that tells towards a very competent throughput [20], [21]. Otherwise,

$$
\text { U Slotted Aloha }(p)=\llbracket N p(1-p) \rrbracket \wedge(N-1)
$$

In Slotted Aloha, a station can transmit successfully with probability at least $1 / e$, or about $36 \%$ of the time where each packets is exactly one time slot long, it holds that:

- More than one node sent in a time slot $=$ collision, wanted time slot. 
- Time slot where exactly one packet is sent $=$ success

- $\quad$ The throughput $=$ uncollided packets per time interval

- $\quad$ The utilization $=$ throughput/channel rate [22]

\subsection{Simple Pure Aloha Algorithm}

Coming up with an algorithm/protocol that enables each node gets reasonable utilization, reasonable fairness and reasonable delay, one way of solving this problem is to calculate the utilization of that protocol as shown in Fig 2 . If each node runs a version of this protocol, and in the protocol each node maintains one variable and the variable it maintains is a probability $\mathrm{p}$ where $\mathrm{p}$ is the probability that a node will transmit a packet if it has a packet to transmit, with each node containing its own variance, therefore if backlogged (waiting processes/packets), packets will be transmitted on the probability p, calculating p we have that: Suppose every node has a value of $\mathrm{p}$, that is $\mathrm{n}$ backlog node, each transmitting with $\mathrm{p}$, how we calculate the utilization, is with the function of $\mathrm{n}$ and $\mathrm{p}$ as shown in Fig. 3. [23], [19]

$$
\mathrm{U}(\mathbf{n}, \mathbf{p})=n p(1-p)^{n-1}
$$

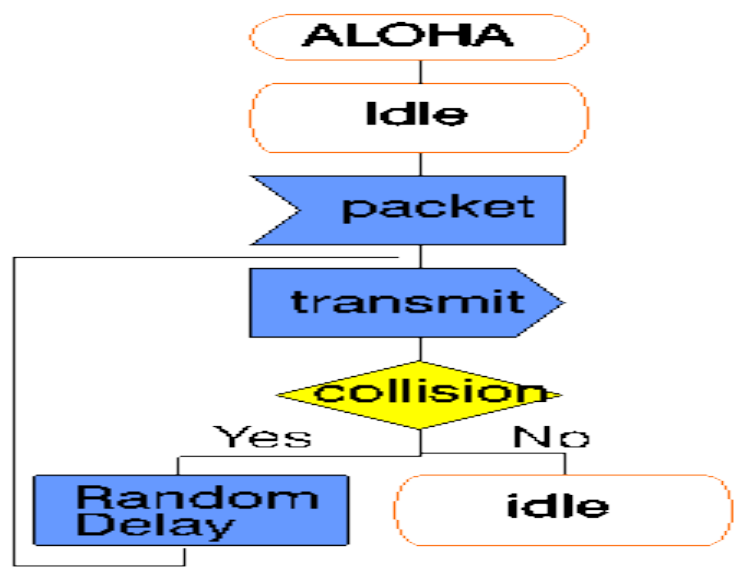

Fig 2: Aloha algorithm for collision check

Where the utilization in this concept is the probability that in any given time there is only one/exactly one successful packet sent, that is the fraction of time slots of exactly one packet.

Throughput $=$ number of successful packet/time slot

$$
\mathbf{p}(1)=p(1-p)^{n-1}, \text { and } U=n \cdot p(1)
$$

Maximum value for $\mathrm{U}$ can be determined by determining the maximum value for $\mathrm{p}$. Let the maximum value for $\mathrm{p}$ be $p^{*}$, then $p^{*}=$ $1 / \mathrm{n}$

Substituting the value of $p^{*}$ into the formula for $\mathrm{U}$ above, we have that the maximum value for $\mathrm{U}$ represented by $U^{*}$ would be given as:

$$
U^{\wedge} *=\quad \| n \cdot(1 / n) \cdot(1-1 / n) \rrbracket^{\wedge}(n-1)=~ \(1-1 / n) \rrbracket^{\wedge}(n-1)
$$

This gives a good utilization that is not zero, as $\mathrm{n}$ tends to infinity the maximum utilization becomes -1 or $1 / \mathrm{e}$.

The basic aloha protocol is using the backlog to check every node sent with the probability $\mathrm{p}$ max which is $1 / \mathrm{n}$

Stabilization: determining at each node the actual value of $\mathrm{p}$ which changes with respect to time at every node. That is if there is a competition amongst nodes $\mathrm{p}$ reduces else $\mathrm{p}$ increases. 
International Journal of Advances in Scientific Research and Engineering (ijasre), Vol 5 (8), August-2019

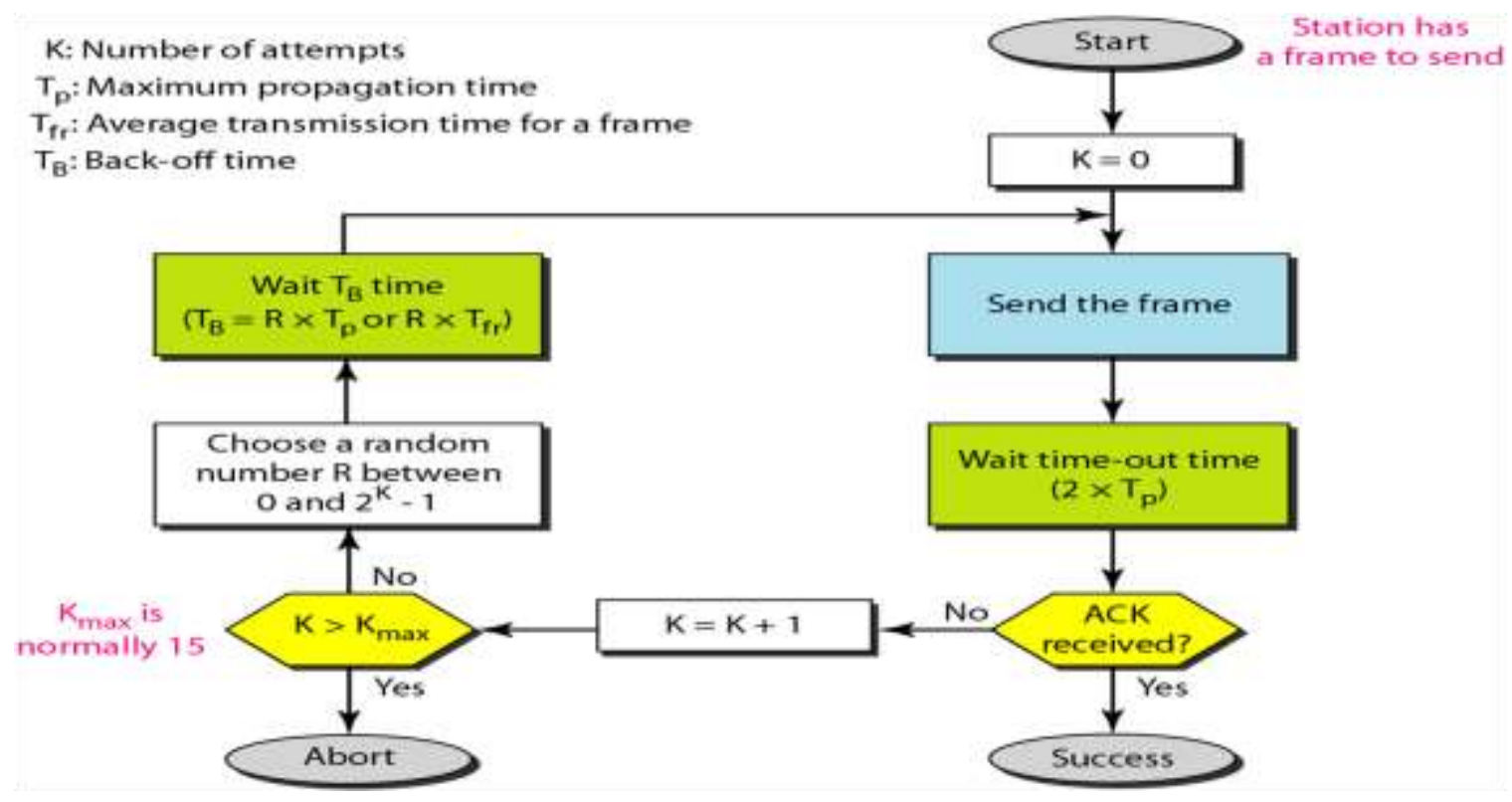

Fig 3: Simple Pure Aloha Algorithm [25]

When a packet is sent, and it fails it gets information by an acknowledgement coming from the receiving node or receiver, some feedback tells the protocol if the packet succeeded or not. When packet don't succeed - collision, what should be done to the nodes transmission probability is to reduce it and one way of doing that is by "multiplicative decrease", that is decreasing by a factor of $2=\mathrm{p} / 2$, when packet succeed - increase transmission $\mathrm{p}$ by doubling it $=2 \mathrm{p}$ [23], [24].

\section{Stabilizing Aloha}

There are four ways in which aloha can be stabilized:

- Modify the protocol by capture effect

- A successful node maintains a very high $\mathrm{p}$ (of average tending to 1 ).

- Starve out other nodes for a short period.

- Try an increase rule with an upper bound node independently making these decisions.

\section{RECOMMENDATION}

Good MAC protocols improves productivity by the means of utilization and fairness and must be able to handle difficulties in an effective and distributed way intended to be experienced to respond to setback in an intelligent way, minimizing collision, delay or waste. In a more practical case, the outlay of an inner central controller node brilliantly aware of which nodes has packets to propel is also high, these protocols must by the cognate token provide useful operation and truthfulness through its utilization under dynamic jade, TDMA provides a relatively high throughput when for the most part of the nodes are accumulated and the offered loads is distributed evenly amongst the receiving nodes. When node/loads are in fragments or different nodes send different amounts of packets individually, TDMA is a underprivileged choice, although Slotted Aloha has a surprisingly high utilization and throughput, if one can select the likelihood exigency at the proper time, the probability that maximizes throughput will be $1 / \mathrm{N}, \mathrm{N}$ is the total number of accumulated nodes, the resulting utilization tends to $1 / \mathrm{e}$ which is equivalent to $37 \%$, and the fairness is end to 1 if for the most part the nodes stand for the same load. The utilization does remains valuable even when the nodes detail different loads, not subordinate to TDMA, so when $\mathrm{N}$ is a large number, these numbers are 1/e and $1-2 / \mathrm{e}$ which are equivalent to $26 \%$, respectively, if $\mathrm{p}$ is reproduced to cut the number of insignificant slots, then we do not increase the utilization but increase the collision rate.

\section{CONCLUSION}

In crowning achievement, stabilization is problematic to making Aloha practical. A format was calculated to adapt the transmitted message likelihood, by decreasing it multiplicatively when a collision occurs and increasing it as a choice multiplicatively or to a fixed maximum number when a transmission occurs. The idea was to seek to gather to the favorable point of value, Slotted Aloha has twice the utilization of Unslotted-Aloha when the numbers of accumulated nodes grows, the insightful reason is that if two packets are fixed to collide, then the "window of vulnerability" is larger in the Unslotted by a factor of two (2). A broadcast network that can use packets that have infinite slots in intensity (i.e., mimicking the Unslotted case) can adopt the carrier sense if the means of transmission is a true broadcast medium or roughly so, in a true broadcast channel, all nodes can listen and hear to each other reliably, so they can sense the carrier once upon transmitting their packets, by "listening before transmitting" and landscape the transmission likelihood via stabilization, they can abbreviate the number of collisions and increase utilization, 
although it is hard for communication nodes not to experience collisions, fairness again requires bounds on the transmission probability as before. A related distribution in a finite window guarantees that each node will stake a transmission within sprinkling fixed number of slots, which is not true for the geometric distribution.

\section{REFERENCES}

1. B. Awerbuch, and A. Mitra, "Media Access Control Protocol (MAC) for Ad Hoc Wireless Network" Advanced Topics in Wireless Network, 2008

2. I. Demirkol, C. Ersoy, and F. Alagoz. "Mac Protocol for Wireless Sensor Networks: A Survey”, 2010, pp1-3.

3. M. Mushaib, P. Rajeev, A.K. Jaiswal, and A. Aditi, "Analyzing and shrinking network inaccessibility in ZIGBEE wireless communication by comparing it with TDMA-MAC protocol." 5th International Conference on Reliability, Infocom Technologies and Optimization (Trends and Future Directions)(ICRITO),2016, pp. 560-565. IEEE.

4. S. Faruque, "Frequency Division Multiple Access (FDMA)." In Radio Frequency Multiple Access Techniques Made Easy, Springer, Cham, 2019, pp. 21-33.

5. M. Perillo, and W. Heinzelman, "Wireless Sensor Network Protocols". Department of Electrical and Computer Engineering , 2012, pp4-6.

6. M. Sharif-Yazd, R.K. Mohammad, and K.M. Mohammad, "A survey on underwater acoustic sensor networks: perspectives on protocol design for signaling, MAC and routing." arXiv preprint arXiv:1703.08353, 2017.

7. S. Cambazoglu, and S. Arif, "Collision Avoidance in Mobile Wireless Ad-Hoc Networks with Enhanced MACAW Protocol Suite." International Journal of Communications, Network and System Sciences, 2015 Volume. 8, Issue 13, pp.533-542.

8. S.K. Sarkar, G.B. Tiptur, and C. Puttamadappa. "Ad hoc mobile wireless networks: principles, protocols, and applications". CRC Press, 2016.

9. A. Djimli, M. Salah, and H. Saad, "Energy-efficient MAC protocols for wireless sensor networks: a survey." TELKOMNIKA, 2019 Volume. 17, Issue 5 pp.2301-2312.

10. A. Sabharwal, "2017 SIGMOBILE Outstanding Contributions Award: Norman Abramson." GetMobile: Mobile Computing and Communications. 2018 Volume. 21, Issue. 4 pp.15-15.

11. M, Richharia, Satellite communication systems: design principles. Macmillan International Higher Education, 2017.

12. M. Jain, D.S. Tomar, and S.K. Singh. "A Survey on TCP Congestion Control Schemes in Guided Media and Unguided Media Communication." International Journal of Computer Applications. 2015 Volume. 118, Issue. 3.

13. Y. Hu, Y. Wang, and K.W.A. Chee. "Optical Communications and Modulation Techniques in 5G." In Smart Grids and Their Communication Systems, Springer, Singapore, 2019, pp. 401-464.

14. P.S. Henry, G.Vannucci, and T.M. Willis III, Apparatus and method for guided wave communications using an absorber. AT\&T Intellectual Property I LP, 2019. U.S. Patent Application 15/828,682.

15. C.R. Stevenson, G. Chouinard, Z. Lei, W. Hu, S.J. Shellhammer, and W. Caldwell. "IEEE 802.22: The first cognitive radio wireless regional area network standard." IEEE communications magazine. 2009, Volume. 47, Issue. 1 pp.130-138.

16. N.J. Karl, R.W. McKinney, Y. Monnai, R. Mendis, and D.M. Mittleman. "Frequency-division multiplexing in the terahertz range using a leaky-wave antenna." Nature Photonics 2015. Volume. 9, Issue. 11 pp.717-725.

17. Z. Yang, Y. Yao, X. Li, and D. Zheng. "A TDMA-based MAC protocol with cooperative diversity." IEEE communications letters. 2010 Volume. 14, Issue. 6 pp.542-544.

18. R. Yadav, S. Varma, and N. Malaviya. "A survey of MAC protocols for wireless sensor networks." UbiCC journal. 2009, Volume. 4, Issue. 3 pp.827-833.

19. Z. Peng, Y. Zhu, Z. Zhou, Z. Guo, and J. Cui. "COPE-MAC: A contention-based medium access control protocol with parallel reservation for underwater acoustic networks." In OCEANS'10 IEEE SYDNEY, IEEE. 2010, pp.1-10. 
20. S. Hu, Y. Yao, and Z. Yang. "MAC protocol identification using support vector machines for cognitive radio networks." IEEE Wireless Communications. 2014 Volume. 21, Issue. 1 pp.52-60.

21. Z. Yibo, Z.J. Peng, M. Zuba, J. Cui, and H. Chen. "Towards Practical MAC Design for Underwater Acoustic Networks". IEEE INFOCOM. 2013.

22. M. Moradian, and F. Ashtiani. "Sum throughput maximization in a slotted Aloha network with energy harvesting nodes." 2014 IEEE Wireless Communications and Networking Conference (WCNC). 2014, pp.1585-1590.

23. P. Šolić, J. Radić, and N. Rožić. "Energy efficient tag estimation method for ALOHA-based RFID systems." IEEE Sensors Journal. 2014 Volume.14, Issue. 10 pp.3637-3647.

24. K. Cohen, A. Leshem, and E. Zehavi. "Game theoretic aspects of the multi-channel ALOHA protocol in cognitive radio networks." IEEE Journal on Selected Areas in Communications. 2013 Volume. 31, Issue. 11 pp.2276-2288.

25. MIT. "Sharing a Channel: Media Access (MAC) Protocols". MIT 0.02 Draft Lecture Note . MIT. 2012. 